\begin{tabular}{|c|l|}
\hline Title & Linear Quadratic Regulator with Decentralized Event-T riggering \\
\hline Author(s) & Nakajima, Kyohei; Kobay ashi, Koichi; Y amashita, Yuh \\
\hline Citation & $\begin{array}{l}\text { IEICE transactions on fundamentals of electronics communications and computer sciences, E100A (2), 414.420 } \\
\text { https://doi.org/10.1587/transfun.E100.A.414 }\end{array}$ \\
\hline Issue Date & 2017-02 \\
\hline Doc URL & http://hdl.handle.net/2115/65212 \\
\hline Rights & copyright@2017 IEICE \\
\hline Type & article \\
\hline File Information & Linear quadratic regulator with decentralized event-triggering.pdf \\
\hline
\end{tabular}

Instructions for use 


\title{
Linear Quadratic Regulator with Decentralized Event-Triggering
}

\author{
Kyohei NAKAJIMA $^{\dagger}$, Nonmember, Koichi KOBAYASHI ${ }^{\dagger a}$, and Yuh YAMASHITA $^{\dagger}$, Members
}

\begin{abstract}
SUMMARY Event-triggered control is a control method that the measured signal is sent to the controller only when a certain triggering condition on the measured signal is satisfied. In this paper, we propose a linear quadratic regulator (LQR) with decentralized triggering conditions. First, a suboptimal solution to the design problem of LQRs with decentralized triggering conditions is derived. A state-feedback gain can be obtained by solving a convex optimization problem with LMI (linear matrix inequality) constraints. Next, the relation between centralized and decentralized triggering conditions is discussed. It is shown that control performance of an LQR with decentralized event-triggering is better than that with centralized event-triggering. Finally, a numerical example is illustrated.

key words: decentralized triggering conditions, event-triggered control, linear matrix inequality, linear quadratic regulator
\end{abstract}

\section{Introduction}

A networked control system (NCS) is a control system where components such as plants, sensors, controllers, and actuators are connected through communication networks. In each component, messages about the control input or the measured output are sent and received (see Fig. 1). During the last decade, there have been a lot of studies on analysis and synthesis of NCSs from several viewpoints such as packet losses, transmission delays, and communication constraints (see, e.g., [1], [4], [11]-[14], [17]).

In the first step of design of NCSs, it is important to select either periodic or aperiodic control methods. In order to decrease the number of sent and received messages, aperiodic control methods are useful. As an aperiodic control method, event-triggered and self-triggered control methods are well known (see e.g., [2], [3], [7]-[10], [15], [16], [18][26]). The basic idea of event-triggered control is that transmissions of the measured signal and the control input are executed, only when a certain triggering condition on the measured signal is satisfied (i.e., the event occurs). The basic idea of self-triggered control is that the next sampling time at which the control input is recomputed is computed based on predictions.

In this paper, we propose a linear quadratic regulator (LQR) with decentralized triggering conditions. The LQR problem is one of the fundamental problems in control theory. A suboptimal solution to the design problem of LQRs

\footnotetext{
Manuscript received April 15, 2016.

Manuscript revised August 16, 2016.

$\dagger$ The authors are with the Graduate School of Information Science and Technology, Hokkaido University, Sapporo-shi, 060-0814 Japan.

a)E-mail: k-kobaya@ssi.ist.hokudai.ac.jp DOI: 10.1587/transfun.E100.A.414
}

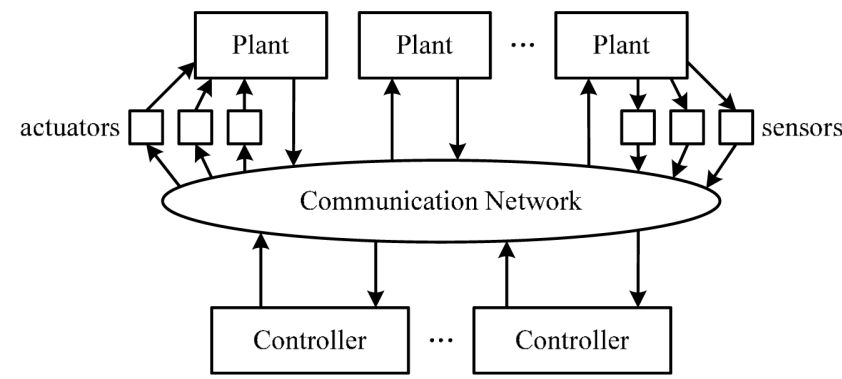

Fig. 1 Illustration of networked control systems. Actuators and sensors may be located in a decentralized way.

with centralized event-triggering has been obtained in [26]. Under the assumption that a triggering condition is given, a state-feedback gain can be obtained by solving a convex optimization problem with LMI (linear matrix inequality) constraints. Decentralized triggering conditions studied here have been proposed in [21]. In this method, each sensor has a certain triggering condition. When a triggering condition of at least one sensor is satisfied, the plant state, which is stored in the controller, is updated (i.e., all sensors send the message about the measured signal to the controller). In the case where sensors are located in a decentralized way, this control method is useful. In [26], decentralized triggering conditions have not been studied.

In this paper, first, a suboptimal solution to the design problem of LQRs with decentralized triggering conditions is derived based on the result in [26]. Then, a state-feedback gain can be obtained by solving a convex optimization problem with LMI constraints. Next, the relation between centralized and decentralized triggering conditions is discussed. We show that although the number of occurrences of events may increase, control performance of an LQR with decentralized triggering is better than that with centralized triggering. Finally, the effectiveness of the proposed method is shown by a numerical example.

The result in this paper provides us a basic result for event-triggered control.

This paper is organized as follows. In Sect. 2, the outline of event-triggered control and triggering conditions is explained, and the design problem of LQRs with decentralized triggering condition is formulated. In Sect. 3, a suboptimal solution to the design problem is derived. In Sect. 4, the relation between centralized and decentralized triggering conditions is discussed. In Sect. 5, a numerical example is presented. In Sect. 6, we conclude this paper. 
Notation: Let $\mathcal{R}$ denote the set of real numbers. Let $I_{n}$, $0_{m \times n}$ denote the $n \times n$ identity matrix, the $m \times n$ zero matrix, respectively. For simplicity, we sometimes use the symbol 0 instead of $0_{m \times n}$, and the symbol $I$ instead of $I_{n}$. For a vector $x$, let $\|x\|$ denote the Euclidean norm of $x$. For a vector $x$, let $x_{i}$ denote the $i$-th element of $x$. For a matrix $M$, let $M^{\top}$ denote the transpose matrix of $M$. For a matrix $M$, let $\operatorname{tr}(M)$ denote the trace of $M$. For matrices $M_{1}, M_{2}, \ldots, M_{n}$, let diag $\left(M_{1}, M_{2}, \ldots, M_{n}\right)$ denote the block-diagonal matrix. The symmetric matrix $\left[\begin{array}{cc}A & B^{\top} \\ B & C\end{array}\right]$ is denoted by $\left[\begin{array}{cc}A & * \\ B & C\end{array}\right]$.

\section{Problem Formulation}

As a plant, consider the following discrete-time linear system:

$$
x(k+1)=A x(k)+B u(k),
$$

where $x(k) \in \mathcal{R}^{n}$ is the state, $u(k) \in \mathcal{R}^{m}$ is the control input, and $k \in\{0,1,2, \ldots\}$ is the discrete time. For the system (1), consider the following event-triggered state-feedback controller:

$$
u(k)=K \hat{x}(k),
$$

where $\hat{x}(k)$ is defined by

$$
\hat{x}(k)=\left\{\begin{array}{l}
x(k) \quad \text { if } u(k) \text { is updated, } \\
\hat{x}(k-1) \quad \text { if } u(k) \text { is not updated, }
\end{array}\right.
$$

where $x(0)(=\hat{x}(-1))$ is given in advance.

In event-triggered control, a triggering condition is given. If a triggering condition is satisfied, then the control input is updated. Here, we explain a centralized triggering condition and a decentralized triggering condition.

First, based on [26], the centralized triggering condition is defined as follows.

Definition 1: The centralized triggering condition is given by

$$
\|\hat{x}(k-1)-x(k)\|>\sigma\|x(k)\|,
$$

where $\sigma>0$ is a given scalar.

Several triggering conditions have been proposed (see, e.g., [9]), but we consider a simple triggering condition. Using the centralized triggering condition, (3) can be rewritten as

$$
\hat{x}(k)=\left\{\begin{array}{l}
x(k) \quad \text { if }\|\hat{x}(k-1)-x(k)\|>\sigma\|x(k)\|, \\
\hat{x}(k-1) \quad \text { if }\|\hat{x}(k-1)-x(k)\| \leq \sigma\|x(k)\| .
\end{array}\right.
$$

Then, the following condition

$$
\|\hat{x}(k)-x(k)\| \leq \sigma\|x(k)\|
$$

is always satisfied.

Next, the decentralized triggering condition is defined as follows.

Definition 2: The decentralized triggering condition is given by

$$
\exists i \in\{1,2, \ldots, n\}\left|\hat{x}_{i}(k-1)-x_{i}(k)\right|>\sigma\left|x_{i}(k)\right|,
$$

where $\sigma>0$ is a given scalar.

The decentralized triggering condition is utilized in the case where sensors are located in a decentralized way. That is, the sensor $i \in\{1,2, \ldots, n\}$ has the triggering condition $\left|\hat{x}_{i}(k-1)-x_{i}(k)\right|>\sigma\left|x_{i}(k)\right|$. If at least one of these triggering conditions is satisfied, then the control input is updated.

Using the decentralized triggering condition, (3) can be rewritten as

$$
\hat{x}(k)=\left\{\begin{array}{l}
x(k) \text { if }(7) \text { holds } \\
\hat{x}(k-1) \quad \text { otherwise. }
\end{array}\right.
$$

Then, the following condition

$$
\left|\hat{x}_{i}(k)-x_{i}(k)\right| \leq \sigma\left|x_{i}(k)\right|, \quad i \in\{1,2, \ldots, n\}
$$

is always satisfied. That is, the condition (6) is always satisfied. Comparing (6) with (9), we see that (9) is a sufficient condition that (6) holds. In other words, if (9) is satisfied, then (6) is satisfied. Conversely, even if (6) is satisfied, then (9) may not be satisfied.

Using the centralized and decentralized triggering conditions, the design problems of linear quadratic regulators (LQRs) are formulated. First, the design problem of LQRs with the centralized triggering condition is given as follows.

Problem 1: For the system (1), suppose that the initial state and the parameter $\sigma>0$ in (4) are given. Then, find an event-triggered state-feedback controller (2) minimizing the following cost function

$$
J=\sum_{k=0}^{\infty}\left\{x^{\top}(k) Q x(k)+u^{\top}(k) R u(k)\right\},
$$

under the centralized triggering condition (5), where $Q \geq 0$ and $R>0$.

A suboptimal solution to this problem can be obtained by solving a convex optimization problem with LMI constraints [26]. The outline will be explained in Sect. 4.

Next, the design problem of LQRs with the decentralized triggering condition is given as follows.

Problem 2: For the system (1), suppose that the initial state and the parameter $\sigma>0 \mathrm{in}$ (7) are given. Then, find an eventtriggered state-feedback controller (2) minimizing the cost function (10) under the decentralized triggering condition (8).

In this paper, this problem is reduced to a convex optimization problem with LMI constraints. By solving it, we can derive a suboptimal solution to Problem 2. 
Remark 1: An LQR with the decentralized triggering condition (8) is a centralized control scheme. Only a triggering condition is decentralized.

\section{Suboptimal Solution to Problem 2}

In order to derive a suboptimal solution to Problem 2, first, Problem 2 is transformed into the problem of minimizing an upper bound of the cost function (10). Next, this problem is transformed into a convex optimization problem with LMI constraints.

\subsection{Transformation of Problem 2}

First, the error variable is defined by $e(k):=\hat{x}(k)-x(k)$. Then, (9) is replaced with

$$
\left|e_{i}(k)\right| \leq \sigma\left|x_{i}(k)\right|, \quad i \in\{1,2, \ldots, n\} .
$$

From $u(k)=K \hat{x}(k)$ and $\hat{x}(k)=x(k)+e(k)$, the closed-loop system can be obtained as

$$
x(k+1)=\Phi x(k)+B K e(k),
$$

where $\Phi=A+B K$.

Next, we introduce the following quadratic Lyapunov function:

$$
V(k)=x^{\top}(k) P x(k),
$$

where $P=P^{\top}>0$. In this paper, according to the result in [26], we consider designing a controller satisfying

$$
\begin{aligned}
& x^{\top}(k+1) P x(k+1)-x^{\top}(k) P x(k) \\
& <-\left\{x^{\top}(k) Q x(k)+u^{\top}(k) R u(k)\right\} .
\end{aligned}
$$

When this condition is satisfied, the closed-loop system (12) is asymptotically stable, that is, $\lim _{k \rightarrow \infty} x(k)=0$ holds. Noting this fact, from (13), we can obtain

$$
\lim _{k \rightarrow \infty} x^{\top}(k) P x(k)-x^{\top}(0) P x(0)=-x^{\top}(0) P x(0)<-J .
$$

Furthermore, we can obtain

$$
J<x^{\top}(0) P x(0)<\operatorname{tr}(P)\|x(0)\|^{2} .
$$

Since the initial state $x(0)$ is given in advance, the problem of minimizing an upper bound of the cost function (10) is reduced to that of minimizing $\operatorname{tr}(P)$.

From the above discussion, the problem of finding a suboptimal solution to Problem 2 is given by the following problem.

Problem 3: For the system (1), suppose that the initial state and the parameter $\sigma>0$ in (11) are given. Then, find a statefeedback gain $K$ minimizing $\operatorname{tr}(P)$ subject to the conditions (11), (12), and (13).

3.2 Reduction to a Convex Optimization Problem with LMI Constraints

Consider transforming Problem 3 into a convex optimization problem with LMI constraints.

First, note $u(k)=K \hat{x}(k)=K(x(k)+e(k))$. Consider substituting (12) and $u(k)=K(x(k)+e(k))$ into (13). Then, the left-hand side of (13) can be obtained as

$$
\begin{aligned}
& x^{\top}(k+1) P x(k+1)-x^{\top}(k) P x(k) \\
&=(\Phi x(k)+B K e(k))^{\top} P(\Phi x(k)+B K e(k)) \\
&-x^{\top}(k) P x(k) \\
&= {\left[\begin{array}{c}
x(k) \\
e(k)
\end{array}\right]^{\top}\left[\begin{array}{cc}
\Phi^{\top} P \Phi-P & * \\
K^{\top} B^{\top} P \Phi & K^{\top} B^{\top} P B K
\end{array}\right]\left[\begin{array}{l}
x(k) \\
e(k)
\end{array}\right] . }
\end{aligned}
$$

The right-hand side can be obtained as

$$
\begin{aligned}
& -\left\{x^{\top}(k) Q x(k)+u^{\top}(k) R u(k)\right\} \\
= & -x^{\top}(k) Q x(k) \\
& -(x(k)+e(k))^{\top} K^{\top} R K(x(k)+e(k)) \\
= & -\left[\begin{array}{c}
x(k) \\
e(k)
\end{array}\right]^{\top}\left[\begin{array}{cc}
Q+K^{\top} R K & * \\
K^{\top} R K & K^{\top} R K
\end{array}\right]\left[\begin{array}{l}
x(k) \\
e(k)
\end{array}\right] .
\end{aligned}
$$

Hence, from (13), we can obtain

$$
\left[\begin{array}{l}
x(k) \\
e(k)
\end{array}\right]^{\top} P_{1}\left[\begin{array}{l}
x(k) \\
e(k)
\end{array}\right]>0
$$

where

$$
\left[\begin{array}{cc}
P-\Phi^{\top} P \Phi-Q-K^{\top} R K & * \\
-K^{\top} B^{\top} P \Phi-K^{\top} R K & -K^{\top} B^{\top} P B K-K^{\top} R K
\end{array}\right] .
$$

From (11), we can obtain

$$
\left[\begin{array}{l}
x(k) \\
e(k)
\end{array}\right]^{\top} P_{2, i}\left[\begin{array}{l}
x(k) \\
e(k)
\end{array}\right] \geq 0, \quad i \in\{1,2, \ldots, n\},
$$

where

$$
\begin{aligned}
P_{2,1} & =\operatorname{diag}(\sigma^{2}, \underbrace{0,0, \ldots, 0,0}_{n-1},-1, \underbrace{0,0, \ldots, 0,0}_{n-1}), \\
P_{2,2} & =\operatorname{diag}(0, \sigma^{2}, \underbrace{0, \ldots, 0,0}_{n-2}, 0,-1, \underbrace{0, \ldots, 0,0}_{n-2}), \\
& \vdots \\
P_{2, n} & =\operatorname{diag}(\underbrace{0,0,0, \ldots, 0}_{n-1}, \sigma^{2}, \underbrace{0,0,0, \ldots, 0}_{n-1},-1) .
\end{aligned}
$$

By applying $\mathcal{S}$-procedure [5] to (15) and (16), we can obtain

$$
P_{1}-\sum_{i=1}^{n} \tau_{i} P_{2, i}>0
$$

where $\tau_{i}>0$ is a scalar decision variable. By defining $X:=\operatorname{diag}\left(\tau_{1}, \tau_{2}, \ldots, \tau_{n}\right), P_{1}-\sum_{i=1}^{n} \tau_{i} P_{2, i}$ is given by

$$
\begin{aligned}
& {\left[\begin{array}{cc}
P-Q-K^{\top} R K-\sigma^{2} X & * \\
-K^{\top} R K & X-K^{\top} R K
\end{array}\right]} \\
& -\left[\begin{array}{c}
\Phi^{\top} \\
K^{\top} B^{\top}
\end{array}\right] P\left[\begin{array}{ll}
\Phi & B K
\end{array}\right] .
\end{aligned}
$$


Next, by applying the Schur complement [5] to (17) with (18), we can obtain

$$
\left[\begin{array}{ccc}
P-Q-K^{\top} R K-\sigma^{2} X & * & * \\
-K^{\top} R K & X-K^{\top} R K & * \\
\Phi & B K & P^{-1}
\end{array}\right]>0 .
$$

Focusing on $\sigma^{2} X$, we use the Schur complement for the above inequality. Then, we can obtain

$$
\left[\begin{array}{cccc}
P-Q-K^{\top} R K & * & * & * \\
-K^{\top} R K & X-K^{\top} R K & * & * \\
\Phi & B K & P^{-1} & * \\
I & 0 & 0 & \frac{1}{\sigma^{2}} X^{-1}
\end{array}\right]>0 .
$$

Furthermore, noting that

$$
\begin{aligned}
& {\left[\begin{array}{cc}
Q+K^{\top} R K & * \\
K^{\top} R K & K^{\top} R K
\end{array}\right]} \\
& =\left[\begin{array}{cc}
Q^{1 / 2} & 0 \\
R^{1 / 2} K & R^{1 / 2} K
\end{array}\right]^{\top}\left[\begin{array}{cc}
Q^{1 / 2} & 0 \\
R^{1 / 2} K & R^{1 / 2} K
\end{array}\right]
\end{aligned}
$$

holds, we can use the Schur complement again, and we can obtain

$$
\left[\begin{array}{cccccc}
P & * & * & * & * & * \\
0 & X & * & * & * & * \\
\Phi & B K & P^{-1} & * & * & * \\
I & 0 & 0 & \frac{1}{\sigma^{2}} X^{-1} & * & * \\
Q^{1 / 2} & 0 & 0 & 0 & I & * \\
R^{1 / 2} K & R^{1 / 2} K & 0 & 0 & 0 & I
\end{array}\right]>0 .
$$

Finally, left-/right-multiplying (19) by the matrix $\operatorname{diag}\left(P^{-1}\right.$, $\left.P^{-1}, I, I, I\right)$, we can obtain

$$
\left[\begin{array}{cccccc}
P^{-1} & * & * & * & * & * \\
0 & P^{-1} X P^{-1} & * & * & * & * \\
\Phi P^{-1} & B K P^{-1} & P^{-1} & * & * & * \\
P^{-1} & 0 & 0 & \frac{1}{\sigma^{2}} X^{-1} & * & * \\
Q^{1 / 2} P^{-1} & 0 & 0 & 0 & I & * \\
R^{1 / 2} K P^{-1} & R^{1 / 2} K P^{-1} & 0 & 0 & 0 & I
\end{array}\right]>0 .
$$

From $\left(X^{-1}-P^{-1}\right)^{\top} X\left(X^{-1}-P^{-1}\right) \geq 0$, the following relation

$$
P^{-1} X P^{-1} \geq 2 P^{-1}-X^{-1}
$$

can be obtained. Hence, a sufficient condition for (20) is given by the inequality in which $P^{-1} X P^{-1}$ in (20) is replaced with $2 P^{-1}-X^{-1}$.

We define $S:=P^{-1}$ and $Y:=X^{-1}$. Noting that $\operatorname{tr}\left(S^{-1}\right)$ is convex [6], we can arrive at the following theorem.

Theorem 1: A suboptimal solution to Problem 3 is obtained by solving the following convex optimization problem with LMI constraints:

Problem 4: Find $S, W$, and $Y$ minimizing $\operatorname{tr}\left(S^{-1}\right)$ subject to the following LMI

$$
\left[\begin{array}{cccccc}
S & * & * & * & * & * \\
0 & 2 S-Y & * & * & * & * \\
A S+B W & B W & S & * & * & * \\
S & 0 & 0 & \frac{1}{\sigma^{2}} Y & * & * \\
Q^{1 / 2} S & 0 & 0 & 0 & I & * \\
R^{1 / 2} W & R^{1 / 2} W & 0 & 0 & 0 & I
\end{array}\right]>0 .
$$

Then, the state-feedback gain $K$ is obtained as $K=W S^{-1}$.

\section{Relation between Centralized and Decentralized Triggering Conditions}

In this section, first, the result [26] in the design problem of LQRs with the centralized triggering condition is explained. Next, the relation between centralized and decentralized triggering conditions is discussed.

\subsection{A Suboptimal Solution to Problem 1}

A suboptimal solution to Problem 1 is explained as a lemma, which has been obtained in [26].

Lemma 1: A suboptimal solution to Problem 1 is obtained by solving the following convex optimization problem with LMI constraints:

Problem 5: Find $S, W$, and $\alpha>0$ minimizing $\operatorname{tr}\left(S^{-1}\right)$ subject to the following LMI

$$
\left[\begin{array}{cccccc}
S & * & * & * & * & * \\
0 & 2 S-\alpha I & * & * & * & * \\
A S+B W & B W & S & * & * & * \\
S & 0 & 0 & \frac{\alpha}{\sigma^{2}} I & * & * \\
Q^{1 / 2} S & 0 & 0 & 0 & I & * \\
R^{1 / 2} W & R^{1 / 2} W & 0 & 0 & 0 & I
\end{array}\right]>0 .
$$

Then, the state-feedback gain $K$ is obtained as $K=W S^{-1}$.

By replacing $Y$ in Problem 4 with $\alpha I$ in Problem 5, Lemma 1 can be obtained. In derivation of Lemma 1 , instead of (11), we consider (6). Then, instead of (16), we can obtain

$$
\left[\begin{array}{l}
x(k) \\
e(k)
\end{array}\right]^{\top} P_{2}\left[\begin{array}{l}
x(k) \\
e(k)
\end{array}\right] \geq 0, \quad P_{2}=\left[\begin{array}{cc}
\sigma^{2} I & 0 \\
0 & -I
\end{array}\right] .
$$

Applying $\mathcal{S}$-procedure to (15) and (22), we can obtain

$$
P_{1}-\tau P_{2}>0 .
$$

where $\tau>0$ is a scalar decision variable. From (23), we can obtain the LMI (21).

\subsection{Discussion}

Even if the decentralized triggering condition (7) is used, (6) must be satisfied. Hence, instead of (11), we may consider (6) as a constraint condition. Then, in design of LQRs with decentralized triggering, the state-feedback gain obtained by 
solving Problem 5 may be used. However, it is not appropriate to apply Problem 5 to design of LQRs with decentralized triggering. Hereafter, we explain it.

First, in the case of using the centralized triggering condition (4), (23) is equivalent to

$$
\left[\begin{array}{c}
x(k) \\
e(k)
\end{array}\right]^{\top}\left(P_{1}-\tau P_{2}\right)\left[\begin{array}{c}
x(k) \\
e(k)
\end{array}\right]>0,
$$

and we can obtain

$$
\left[\begin{array}{l}
x(k) \\
e(k)
\end{array}\right]^{\top} P_{1}\left[\begin{array}{l}
x(k) \\
e(k)
\end{array}\right]-\tau \underbrace{\left(\sigma^{2} \sum_{i=1}^{n} x_{i}^{2}(k)-\sum_{i=1}^{n} e_{i}^{2}(k)\right)}_{\sigma^{2}\|x(k)\|^{2}-\|e(k)\|^{2}}>0 .
$$

From this expression, we see that in (23), the condition (6) is directly included. In the case of using the decentralized triggering condition (7), (17) is equivalent to

$$
\left[\begin{array}{l}
x(k) \\
e(k)
\end{array}\right]^{\top}\left(P_{1}-\sum_{i=1}^{n} \tau_{i} P_{2, i}\right)\left[\begin{array}{l}
x(k) \\
e(k)
\end{array}\right]>0
$$

and we can obtain

$$
\left[\begin{array}{l}
x(k) \\
e(k)
\end{array}\right]^{\top} P_{1}\left[\begin{array}{l}
x(k) \\
e(k)
\end{array}\right]-\underbrace{\left(\sigma^{2} \sum_{i=1}^{n} \tau_{i} x_{i}^{2}(k)-\sum_{i=1}^{n} \tau_{i} e_{i}^{2}(k)\right)}_{\sigma^{2}\|T x(k)\|^{2}-\|T e(k)\|^{2}}>0,
$$

where $T=\operatorname{diag}\left(\tau_{1}^{1 / 2}, \tau_{2}^{1 / 2}, \ldots, \tau_{n}^{1 / 2}\right)$. From this expression, we see that in (17), the condition (11) is not directly included. In other words, $x(k)$ and $e(k)$ are scaled by the matrix $T$. Comparing (24) with (25), we see that (24) is the special case of (25). That is, by imposing the constraint $\tau_{1}=\tau_{2}=$ $\cdots=\tau_{n}(=: \tau),(25)$ can be rewritten as (24). Hence, in the case of using Theorem 1 and Lemma 1, control performance of an LQR with decentralized triggering is better than that with centralized triggering. Thus, in the case of using the decentralized triggering condition (7), applying Problem 4 is appropriate.

We remark here that the number of times that the decentralized triggering condition (7) is satisfied may be more than the number of times that the centralized triggering condition (4) is satisfied. However, in the case where sensors are located in a decentralized way, the decentralized triggering condition is useful for decreasing the number of communications. When the centralized triggering condition is applied to this case, a certain sensor (or the controller) must aggregate all elements of the state from other sensors, and communications occur at each time.

\section{Numerical Example}

As an example, consider the following discrete-time linear system:

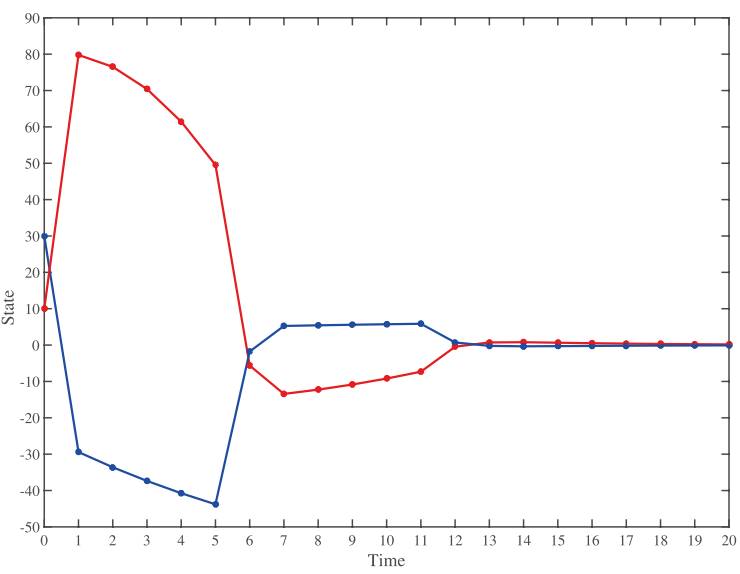

Fig. 2 State trajectory.

$$
x(k+1)=\left[\begin{array}{cc}
1.1 & 0.6 \\
0 & 0.9
\end{array}\right] x(k)+\left[\begin{array}{c}
0.9 \\
-1.0
\end{array}\right] u(k) .
$$

For this system, we suppose that $Q, R$, and $x(0)$ are given by $Q=10 I_{2}, R=1$, and [ $\left.\begin{array}{ll}10 & 30\end{array}\right]^{\top}$, respectively. The parameter $\sigma$ in the decentralized triggering condition (7) is given by $\sigma=0.2$.

By solving Problem 4, we can obtain

$$
\begin{aligned}
S & =\left[\begin{array}{cc}
0.0092 & -0.0060 \\
-0.0060 & 0.0048
\end{array}\right], \\
W & =\left[\begin{array}{ll}
-0.0035 & 0.0037
\end{array}\right], \\
Y & =\left[\begin{array}{cc}
0.0015 & 0 \\
0 & 0.0004
\end{array}\right] .
\end{aligned}
$$

The state-feedback gain $K$ can be obtained as

$$
K=W S^{-1}=\left[\begin{array}{ll}
0.6972 & 1.6484
\end{array}\right] .
$$

Figure 2 and Fig. 3 show the state trajectory and the control input, respectively. From Fig. 2, we see that the state converges to the origin. Figure 4 shows the event, where " 1 " implies the event occurs (i.e., the triggering condition is satisfied) and " 0 " implies the event does not occur. From Fig. 4, we see that the event does not occur at time 2, 3, 4, 8, 9 , and 10 .

Finally, we compare the proposed method with existing methods. In this example, an LQR with centralized triggering cannot be obtained. That is, Problem 5 was infeasible, because the condition (24) is stricter than the condition (25). Hence, we compare the proposed method with the conventional LQR for discrete-time linear systems. First, we discuss the optimality. In this example, the optimal value of the cost function $\operatorname{tr}\left(S^{-1}\right)\|x(0)\|^{2}$ in Problem 4 was derived as $\operatorname{tr}\left(S^{-1}\right)\|x(0)\|^{2}=1.48 \times 10^{6}$. On the other hand, the optimal value of the cost function in the conventional LQR was derived as $x^{\top}(0) P_{d} x(0)=2.58 \times 10^{5}$, where $P_{d}$ is the positive-definite solution of discrete-time algebraic Riccati equation, and was obtained as

$$
P_{d}=\left[\begin{array}{ll}
114.3021 & 134.5739 \\
134.5739 & 184.4115
\end{array}\right] .
$$




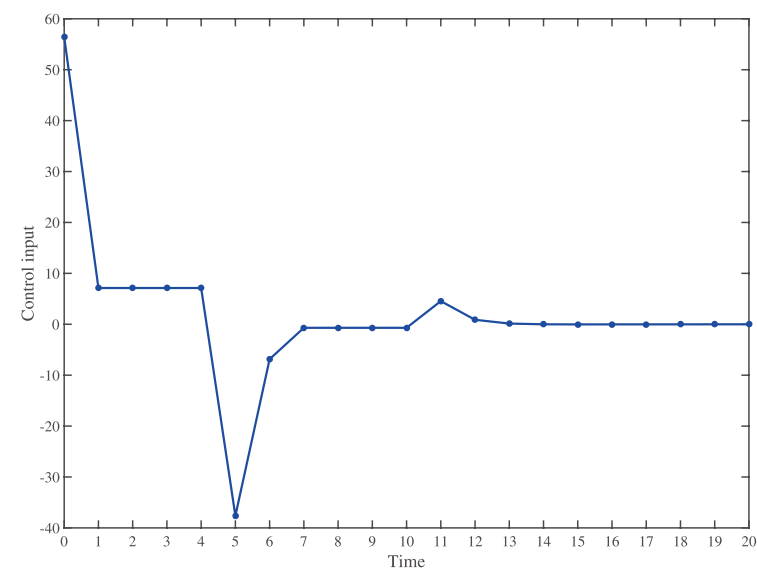

Fig. 3 Control input.

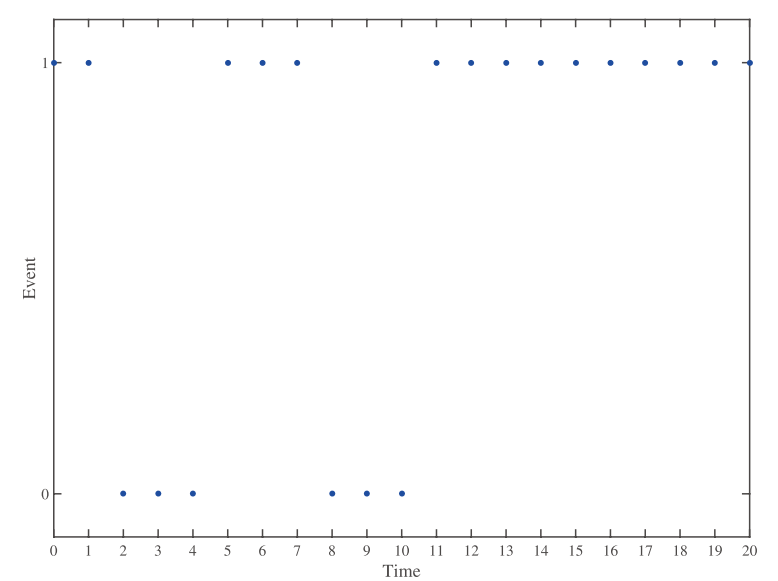

Fig. 4 Event trajectory. "1" implies the event occurs (i.e., the triggering condition is satisfied). "0" implies the event does not occur.

Furthermore, the value of the cost function (10) can be directly calculated from Fig. 2 and Fig. 3. Then, we can obtain $J=3.26 \times 10^{5}$. Comparing these three values, we see that $\operatorname{tr}\left(S^{-1}\right)\|x(0)\|^{2}$ is conservative, but the actual deterioration of the cost function is about $+26 \%$. Next, we discuss the number of times of transmissions. We focus on the time interval $[0,20]$. In the conventional $\mathrm{LQR}$, at time 0 , the message about the control input is sent to the plant. At time $k(k \neq 0)$, two sensors transmit the message about the measured signal to the controller. In addition, the controller transmits the message about the control input to the plant. From these facts, the number of times of transmission at time $k(k \neq 0)$ is 3 . Hence, the number of times of transmissions in the time interval $[0,20]$ is $1+3 \times 20=61$. In the case of using the LQR with decentralized triggering, when the event occurs, sensor 1 (or 2) transmits the message about measured $x_{1}$ (or $x_{2}$ ) to sensor 2 (or 1). After that, sensor 2 (or 1) transmits the message about measured $x_{1}$ and $x_{2}$ to the controller. The controller transmits the message about the updated control input to the plant. Hence, from Fig. 4, the number of times of transmissions is $1+3 \times 14=43$. Thus, the number of times of transmissions can decrease by using
LQRs with decentralized triggering.

\section{Conclusion}

In this paper, we considered the design problem of LQRs with decentralized triggering conditions. First, a suboptimal solution to this problem was derived. Next, the relation between centralized and decentralized triggering conditions was discussed from the viewpoint of control performance, Finally, a numerical example was presented. The result in this paper provides us a basic result for event-triggered control.

One of the future efforts is to reduce conservativeness. For example, in (14), $\operatorname{tr}(P)$ may be replaced with the maximum eigenvalue of $P$. Further discussion is needed. Optimizing the parameter $\sigma$ in triggering conditions is also an open problem. Finally, in the decentralized triggering condition studied here, when the decentralized triggering condition (8) is satisfied, all elements of the state must be sent to the controller. However, it is desirable that only elements that a triggering condition is satisfied are sent to the controller, and the control input is updated by using only sent elements. This topic has been studied in [22], [24], but the case of LQRs has not been studied. This topic is also one of the future efforts.

This work was partly supported by JSPS KAKENHI Grant Numbers 26420412, 16H04380.

\section{References}

[1] C.T. Abdallah and H.G. Tanner, "Complex networked control systems: Introduction to the special section," IEEE Control Syst. Mag., vol.27, no.4, pp.30-32, 2007.

[2] A. Anta and P. Tabuada, "Self-triggered stabilization of homogeneous control systems," Proc. 2008 American Control Conf., pp.4129-4134, 2008.

[3] A. Anta and P. Tabuada, "To sample or not to sample: Self-triggered control for nonlinear systems," IEEE Trans. Autom. Control, vol.55, no.9, pp.2030-2042, 2010.

[4] P. Antsaklis and J. Baillieul, "Special issue on technology of networked control systems," Proc. IEEE, vol.95, no.1, pp.5-8, 2007.

[5] S. Boyd, L. El Ghaoul, E. Feron, and V. Balakrishnan, Linear Matrix Inequalities in System and Control Theory, SIAM, 1997.

[6] S. Boyd and L. Vandenberghe, Convex Optimization, Cambridge University Press, 2004

[7] A. Camacho, P. Martí, M. Velasco, C. Lozoya, R. Villa, J.M. Fuertes, and E. Griful, "Self-triggered networked control systems: An experimental case study," Proc. IEEE Int'l Conf. Industrial Technology, pp.123-128, 2010.

[8] A. Girard, "Dynamic triggering mechanisms for event-triggered control,” IEEE Trans. Autom. Control, vol.60, no.7, pp.1992-1997, 2015.

[9] W.P.M.H. Heemels, K.H. Johansson, and P. Tabuada, "An introduction to event-triggered and self-triggered control," Proc. 51st IEEE Conf. Decision and Control, pp.3270-3285, 2012.

[10] W.P.M.H. Heemels, M.C.F. Donkers, and A.R. Teel, "Periodic eventtriggered control for linear systems," IEEE Trans. Autom. Control, vol.58, no.4, pp.847-861, 2013.

[11] L.-S. Hu, T. Bai, P. Shi, and Z. Wu, "Sampled-data control of networked linear control systems," Automatica, vol.43, no.5, pp.903911, 2007.

[12] H. Ishii, "Stabilization under shared communication with message 
losses and its limitations," Proc. 45th IEEE Conf. Decision and Control, pp.4974-4979, 2006.

[13] H. Ishii, " $H^{\infty}$ control with limited communication and message losses," Syst. Control Lett., vol.57, no.4, pp.322-331, 2008.

[14] K. Kobayashi and K. Hiraishi, "Mixed-integer-programming-based approach to optimal design of networked control systems," SICE Journal of Control, Measurement, and System Integration, vol.4, no.3, pp.243-248, 2011.

[15] K. Kobayashi and K. Hiraishi, "Self-triggered model predictive control with delay compensation for networked control systems," IEICE Trans. Fundamentals, vol.E96-A, no.5, pp.861-868, 2013.

[16] K. Kobayashi and K. Hiraishi, "Self-triggered model predictive control using optimization with prediction horizon one," Math. Probl. Eng., vol.2013, article ID 916040, 9 pages, 2013.

[17] K. Kobayashi and K. Hiraishi, "An optimization-based approach to sampled-data control of networked control systems with multiple delays," Appl. Math. Comput., vol.247, pp.786-794, 2014.

[18] K. Kobayashi and K. Hiraishi, "Event-triggered and self-triggered control for networked control systems using online optimization,' IEICE Trans. Fundamentals, vol.E99-A, no.2, pp.468-474, 2016.

[19] D. Lehmann, E. Henriksson, and K.H. Johansson, "Event-triggered model predictive control of discrete-time linear systems subject to disturbances, Proc. 2013 European Control Conf., pp.1156-1161, 2013

[20] M. Mazo, Jr. and P. Tabuada, "On event-triggered and self-triggered control over sensor/actuator networks," Proc. 47th IEEE Conf. Decision and Control, pp.435-440, 2008.

[21] M. Mazo, Jr. and P. Tabuada, "Decentralized event-triggered control over wireless sensor/actuator networks," IEEE Trans. Autom. Control, vol.56, no.10, pp.2456-2461, 2011.

[22] M. Mazo, Jr. and M. Cao, "Asynchronous decentralized eventtriggered control," Automatica, vol.50, no.12, pp.3197-3203, 2014.

[23] S. Nakao and T. Ushio, "Self-triggered predictive control with timedependent activation costs of mixed logical dynamical systems," IEICE Trans. Fundamentals, vol.E97-A, no.2, pp.476-483, 2014.

[24] P. Tallapragada and N. Chopra, "Decentralized event-triggering for control of LTI systems," Proc. 2013 IEEE Int'1 Conf. Control Applications, pp.698-703, 2013.

[25] X. Wang and M.D. Lemmon, "Self-triggered feedback control systems with finite-gain $\mathcal{L}_{2}$ stability," IEEE Trans. Autom. Control, vol.54, no.3, pp.452-467, 2009.

[26] W. Wu, S. Reimann, D. Görges, and S. Liu, "Suboptimal eventtriggered control for time-delayed linear systems," IEEE Trans. Autom. Control, vol.60, no.5, pp.1386-1391, 2015.

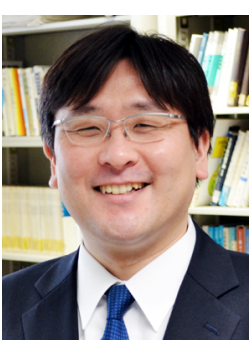

Koichi Kobayashi received the B.E. degree in 1998 and the M.E. degree in 2000 from Hosei University, and the D.E. degree in 2007 from Tokyo Institute of Technology. From 2000 to 2004 he worked at Nippon Steel Corporation. From 2007 to 2015, he was an Assistant Professor at Japan Advanced Institute of Science and Technology. Since 2015, he has been an Associate Professor at the Graduate School of Information Science and Technology, Hokkaido University. His research interests include analysis and control of discrete event and hybrid systems. He is a member of the SICE, ISCIE, IEEJ, and IEEE.

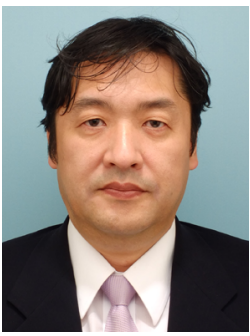

Yuh Yamashita received his B.S., M.S., and Ph.D. degrees from Hokkaido University, Japan, in 1984, 1986, and 1993, respectively. In 1988, he joined the faculty of Hokkaido University. From 1996 to 2004, he was an Associate Professor at the Nara Institute of Science and Technology, Japan. Since 2004, he has been a Professor of the Graduate School of Information Science and Technology, Hokkaido University. His research interests include nonlinear control and nonlinear dynamical systems. He is a mem-

ber of SICE, ISCIE, SCEJ, and IEEE.

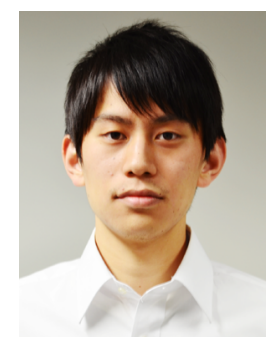

Kyohei Nakajima received the B.E. degree in 2016 from Hokkaido University. Since 2016, he has been pursuing the M.E. degree with the Graduate School of Information Science and Technology, Hokkaido University. His research interests include networked control systems. 